\title{
Neue Weinkunden-Segmentierung in Deutschland
}

\author{
Gergely Szolnoki and Dieter Hoffmann \\ Geisenheim University, Department of Business Administration and Market Research, Von-Lade-Str. 1, \\ 65366 Geisenheim, Germany
}

\begin{abstract}
Unabhängig von der Branche war es immer schon von großer Bedeutung, Kundenwünsche zu kennen und die Kunden zu verstehen. Die Methode der Segmentierung bietet eine gute Möglichkeit, diesen Anforderungen gerecht zu werden. Sie verdeutlicht das Kaufverhalten von Kunden und bündelt diese in homogene Gruppen. Diese Studie basiert auf einer repräsentativen Befragung, die in 2012 mit 2000 Teilnehmern in Deutschland durchgeführt wurde. Ziel der Befragung war es, den deutschen Weinmarkt mit einer neuen Methode zu modellieren und dabei unterschiedliche Verbrauchersegmente zu bilden. Insgesamt wurden neun Segmentierungsmodelle entwickelt: Deutsche Weinkonsumenten wurden nach Geschlecht, Alter, Region, Sozialen Klassen, Konsumintensität, Geschmackspräferenz, Farbpräferenz, Herkunftspräferenz und Nutzung von Einkaufsstätten aufgeteilt und deren verhaltensbezogene Eigenschaften detailliert analysiert. Die Ergebnisse zeigen die Nützlichkeit dieser Art von Segmentierung und beschreiben den deutschen Weinmarkt sowohl mengen- als auch wertmäßig.
\end{abstract}

\section{Einleitung}

In der Weinbranche mangelt es nicht an Segmentierungsmodellen. Es stellt sich allerdings die Frage, wie gut diese Modelle die Marktrealität beschreiben.

Ein kleines Forschungsteam des Instituts für Betriebswirtschaft und Marktforschung setzte sich vor ein paar Jahren das Ziel, Segmentierungsmodelle für die Weinbranche zu entwickeln, die es ermöglichen, den Markt und das Verhalten von Weinkonsumenten realitätsnah zu beschreiben. Dieser Artikel gibt einen kleinen Überblick über die bisher veröffentlichten Studien sowie über die Ergebnisse des Geisenheimer Segmentierungsprojektes und zwei drei Beispiele der WeinkundenSegmentierung.

\section{Literaturüberblick}

Obwohl vor zehn Jahren Autoren wie Thomas \& Pickering (2003) und Hughson et al. (2004) noch darüber berichteten, dass die Segmentierung in der Weinbranche schwach entwickelt sei und bisher wenig über dieses Thema publiziert wurde, können wir heute genau das Gegenteil feststellen. In der Weinbranche findet man derzeit eine Vielzahl von Segmentierungen. Wissenschaftler - sowohl in der Alten als auch in der Neuen Welt - veröffentlichen jährlich mehrere Studien mit immer neuen Ansätzen und Segmenten.

Wie Tabelle 1 zeigt, sind die Studien sehr heterogen und bewegen sich auf einer breiten Skala. Die ersten Segmentierungsstudien in den 90er Jahren basierten noch auf Kaufmotiven und Konsumeigenschaften, während spätere Modelle mit völlig anderen Segmentierungskriterien operierten. Verhaltensbezogene und soziodemographische Eigenschaften gehören $\mathrm{zu}$ den meistverwendeten Eigenschaften, die für die Segmentierung herangezogen wurden.
Involvement- bzw. Lifestyle-basierte Segmentierungen wurden zuerst in Australien durchgeführt und später auch in Ländern der Alten Welt eingesetzt. In Deutschland hat die Sinus Studie mithilfe der Lifestyle-Methode ein Segmentierungsmodell entwickelt, das jedoch bestimmte Schwächen aufwies: i) die Segmente wurden auf Basis von qualitativen Gruppendiskussionen entwickelt; ii) man stößt auf Schwierigkeiten, wenn es darum geht, die in der Studie beschriebenen Segmente auf dem Markt zu finden.

Für eine erfolgreiche Segmentierung werden im Idealfall repräsentative Studien verwendet. Aber auch wenn es auf einer repräsentativen Studie basiert, wird am Ende immer nur ein Segmentierungsmodell ausgearbeitet. Dieser reicht aber nicht aus, um das Konsumentenverhalten in allen Details analysieren zu können. Aus diesem Grunde hat unser Team mehrere Segmentierungsmodelle aus derselben repräsentativen Datenbasis entwickelt. Je nachdem, welche Kriterien Produzenten oder Händler zur Analyse einer Konsumentengruppe heranziehen möchten, können sie einen Ansatz oder mehrere Herangehensweisen auswählen. In der Studie wurden Segmentierungen nach folgenden Kriterien durchgeführt: Geschlecht, Alter, Bundesland, Trinkhäufigkeit, Involvement (Interesse und Wissen), Geschmackspräferenz, Farbpräferenz, Herkunftspräferenz, soziale Klasse, soziale Klasse mit Herkunftspräferenz und Einkaufsstätten.

\section{Vorgehensweise}

Beispielhaft zeigen wir hier einige ausgewählte Ergebnisse aus drei Segmentierungen, die nach Trinkhäufigkeit, sozialer Lage sowie Nutzung der Einkaufsstätte ausgewertet wurden. Grundlage der folgenden Auswertungen und der dargestellten Erkenntnisse über die Verbrauchersegmentierung ist eine nach soziodemographischen Kriterien repräsentative Befragung von Männern und Frauen im Alter ab 16 Jahren in der Bundesrepublik Deutschland.

This is an Open Access article distributed under the terms of the Creative Commons Attribution License 4.0, which permits unrestricted use, distribution, and reproduction in any medium, provided the original work is properly cited. 
Tabelle 1. Überblick der Segmentierungsstudien.

\begin{tabular}{|c|c|c|}
\hline Autoren & Land & \begin{tabular}{|l} 
Basis der \\
Segmentierung
\end{tabular} \\
\hline McKinna (1987) & AUS & Kauf und Konsum \\
\hline Spawton (1991) & AUS & Kauf und Konsum \\
\hline Hall and Winchester (1999) & AUS & Kauf und Konsum \\
\hline Dubow (1992) & USA & Anlass \\
\hline Hall et al. (1997) & AUS & Anlass \\
\hline Berni et al. (2005) & DK & Anlass \\
\hline Johnson et al. (1991) & AUS & Verhaltensbezog. \\
\hline Thomas and Pickering (2003) & $\mathrm{NZ}$ & $\begin{array}{l}\text { Verhaltensbezog. } \\
\text { \& Soziodemo. }\end{array}$ \\
\hline Casini et al. (2006) & ITA & Verhaltensbezog. \\
\hline Goodman et al. (2008) & $\begin{array}{l}\text { AUS/ } \\
\text { ISL }\end{array}$ & $\begin{array}{l}\text { Konsum, } \\
\text { Involvement und } \\
\text { Soziodem. }\end{array}$ \\
\hline Lockshin and Cohen (2009) & 11 Land & Verhaltensbezog. \\
\hline Kalazić et al. (2010) & HR & Verhaltensbezog. \\
\hline Mueller and Szolnoki (2010) & $\mathrm{D}$ & Verhaltensbezog. \\
\hline Riviezzo et al. (2011) & ITA & Verhaltensbezog. \\
\hline Quester and Smart (1996) & AUS & Involvement \\
\hline Lockshin et al. (1997) & AUS & Involvement \\
\hline Lockshin et al. (2001) & $\begin{array}{l}\text { AUS/ } \\
\text { FRA }\end{array}$ & Involvement \\
\hline Aurifeille et al. (2002) & $\begin{array}{l}\text { AUS/ } \\
\text { FRA }\end{array}$ & Involvement \\
\hline Hall et al. (2004) & AUS & Soziodem. \\
\hline McGarry et al. (2005) & USA & Soziodem. \\
\hline Thach and Olsen (2006) & USA & Soziodem. \\
\hline Olsen et al. (2007) & USA & Soziodem. \\
\hline Fountain and Lamb (2011) & $\mathrm{NZ}$ & Soziodem. \\
\hline Mueller et al. (2011) & \begin{tabular}{|l|}
5 \\
Land
\end{tabular} & Soziodem. \\
\hline Agnoli et al. (2011) & ITA & Soziodem. \\
\hline McKinna (1987) & AUS & Lifestyle \\
\hline Hall and Winchester (1999) & AUS & Lifestyle \\
\hline Bruwer et al. (2002) & AUS & Lifestyle \\
\hline Johnson and Bruwer (2003) & AUS & Lifestyle \\
\hline Bruwer and Li (2007) & AUS & Lifestyle \\
\hline Hofmeister and Totth (2003) & HUN & Lifestyle \\
\hline Geraghty and Torres (2009) & ISL & Lifestyle \\
\hline Brunner and Siegrist (2011) & $\mathrm{CH}$ & Lifestyle \\
\hline Simpson and Bretherton (2004) & $\mathrm{NZ}$ & Weintourismus \\
\hline Kolyesnikova et al. (2008) & USA & Regionale Weine \\
\hline Goodman et al. (2010) & AUS & Einkaufsstätten \\
\hline Alebaki and Iakovidou (2010) & GRE & Weintourismus \\
\hline
\end{tabular}

Deren Gesamtheit beträgt 65,7 Millionen Personen der Wohnbevölkerung in Privathaushalten. Daraus wurde eine repräsentative Stichprobe im Umfang von 2068 Personen gezogen. Die Befragung erfolgte im Rahmen einer Quotenstichprobe und wurde mithilfe eines strukturierten Fragebogens von den Mitarbeitern der Gesellschaft für Konsumforschung (Omnibusservice) in Nürnberg an 504 verschiedenen Standorten durchgeführt.

\section{Ergebnisse}

\subsection{Segmentierung nach Konsumintensität}

Es liegt nahe, dass Konsumenten, die täglich Wein trinken, ganz andere Präferenzen und Prioritäten haben, als Gelegentlich- oder Wenig-Trinker.
Tabelle 2. Bedeutung der Intensitäts-Segmente im Weinmarkt.

\begin{tabular}{|l|c|c|c|c|}
\hline & $\begin{array}{c}\text { Personen- } \\
\text { anteil }\end{array}$ & $\begin{array}{c}\text { Mengen- } \\
\text { anteil }\end{array}$ & $\begin{array}{c}\text { Wert- } \\
\text { anteil }\end{array}$ & $\begin{array}{c}\text { Durch- } \\
\text { schnitts-preis }\end{array}$ \\
\hline Intensiv & $24 \%$ & $72 \%$ & $75 \%$ & $4,99 € / 1$ \\
\hline Gelegentlich & $38 \%$ & $22 \%$ & $21 \%$ & $4,42 € / 1$ \\
\hline Selten & $38 \%$ & $6 \%$ & $5 \%$ & $3,97 € / 1$ \\
\hline
\end{tabular}

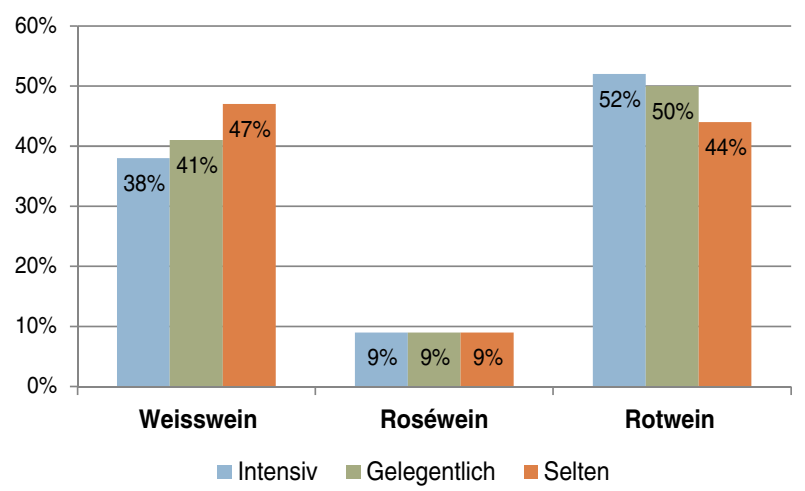

Abb 1. Zusammenhang zwischen Weinfarbe und Konsumintensität.

Die Häufigkeiten haben gezeigt, dass sich die Gruppe der "Intensiv-Verbraucher" aus denjenigen Personen zusammensetzt, die mehrmals pro Woche und mindestens einmal pro Woche Wein trinken. Demgegenüber kennzeichnete die Gruppe der "Selten-Verbraucher", dass sie seltener als einmal pro Monat Wein konsumiert. Als "Gelegentlich-Verbraucher" sind diejenigen zu bezeichnen, die 2-bis 3-mal pro Monat oder einmal pro Monat Wein trinken. Daraus ließ sich ableiten, dass auch die gelegentlichen Weintrinker mehr oder weniger regelmäßige Weinkonsumenten sind.

Hinsichtlich des Personenanteils bildeten die "IntensivVerbraucher" das kleinste Segment, aber deren Mengenund Wertanteile wiesen mit 3/4 des Gesamtmarktes auf die große Bedeutung dieser Kunden hin (Tabelle 2).

Die Auswertung der Segmente nach Konsumintensität zeigte interessante Zusammenhänge. Zum einen wurde deutlich, dass der größte Teil des Weinkonsums von einer relativ kleinen Gruppe von mehr oder weniger regelmäßig konsumierenden Verbrauchern getätigt wird. Diese zeigten in Verbindung mit einer besseren Ausbildung und einem höheren sozialen Status mehr Interesse an Wein und sie verwendeten weinspezifische Kriterien für ihre Kaufentscheidung viel differenzierter als andere Gruppenangehörige.

Abb. 1 zeigt den Zusammenhang zwischen Weinfarbe und Konsumintensität. Je häufiger die Konsumenten Wein tranken, desto höher lag der Rotwein-Anteil. Da Trinkhäufigkeit und Weininteresse bzw. Weinkenntnis stark miteinander korrelieren, kann man weitergehend schlussfolgern, dass Konsumenten, die ein hohes Involvement aufzeigen und regelmäßig Wein trinken, häufiger Rotwein als der Durchschnitt zu sich nehmen.

\subsection{Segmentierung nach Sozialer Lage}

In vielen Studien werden soziodemographische Verbraucherkriterien nicht mehr als hilfreich für die Differenzierung der Konsumenten in verschiedene Segmente 
Tabelle 3. Bedeutung der sozialen Lage im Weinmarkt.

\begin{tabular}{|l|c|c|c|c|}
\hline & $\begin{array}{c}\text { Personen- } \\
\text { anteil }\end{array}$ & $\begin{array}{c}\text { Mengen- } \\
\text { anteil }\end{array}$ & $\begin{array}{c}\text { Wert- } \\
\text { anteil }\end{array}$ & $\begin{array}{c}\text { Durch- } \\
\text { schnitts-preis }\end{array}$ \\
\hline A-Weinkunden & $33 \%$ & $43 \%$ & $47 \%$ & $4,93 € / l$ \\
\hline B-Weinkunden & $47 \%$ & $30 \%$ & $30 \%$ & $4,33 € / 1$ \\
\hline C-Weinkunden & $20 \%$ & $27 \%$ & $23 \%$ & $3,74 € / l$ \\
\hline
\end{tabular}

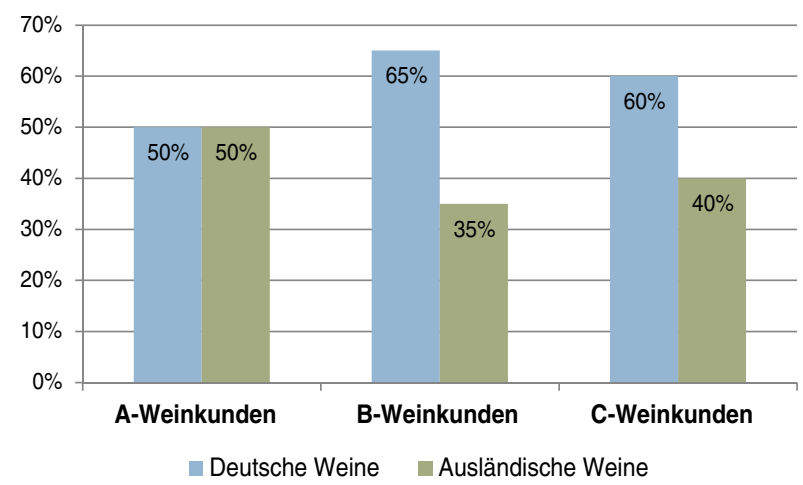

Abb 2. Herkunftspräferenz der A-, B- und C-Weinkunden.

angesehen. Aus diesem Grund wurde analysiert, ob soziodemographische Kriterien einen Einfluss auf das Verbraucherverhalten bezüglich Wein haben und welche weinspezifischen Orientierungen die Vertreter einzelner Segmente aufwiesen.

Werden die Weinkonsumenten nach sozialen Gruppen (z.B. A-Weinkunden = Oberschicht) differenziert, so wurde deutlich, dass der Umfang und die Struktur des Weinverbrauchs sehr wohl von der sozialen Lage beeinflusst werden. Die Auswertung der Weinkonsumenten nach Segmenten mit unterschiedlicher sozialer Lage lieferte tiefere Einblicke in die Weinkundenstrukturen und deren Präferenzen. In der "A-Kundengruppe" wurde mehr und hochpreisiger Wein konsumiert (Tabelle 3). Damit konnte gezeigt werden, dass der Weinverbrauch eher in der Oberschicht angesiedelt und demzufolge weniger konjunkturabhängig ist.

Die "A-Weinkunden" tranken lediglich zu 50\% deutsche Weine, obwohl sie verstärkt bei Winzern einkauften. Dies bedeutete im Umkehrschluss, dass ausgerechnet die von der Wertschöpfung her wichtigste Konsumentengruppe signifikant weniger einheimischen Wein konsumiert und die Deutschweintrinker eher in den Konsumentengruppen $\mathrm{B}$ und $\mathrm{C}$ zu finden sind.

\subsection{Einkaufsstätten-Segmentierung}

Im Rahmen dieser Segmentierung wurde auch die Nutzung der Einkaufsstätten von Konsumenten untersucht. Gemäß der Studie konnten sechs verschiedene Verbrauchergruppen hinsichtlich der Nutzung unterschiedlicher Einkaufsstätten sowie der Kaufhäufigkeit von Wein definiert werden.

Tabelle 4 zeigt, wie unterschiedlich die Segmente sind, wenn es um Größe, Mengenanteil oder Wertanteil der Gruppen geht. Die Auswertung machte deutlich, dass auch kleinere Nischen-Gruppen, wie z. B. FachgeschäftKunden, eine sehr wichtige Rolle bei der Wertschöpfung spielten.
Tabelle 4. Bedeutung der Einkaufsstätten-Segmente im Weinmarkt.

\begin{tabular}{|l|c|c|c|c|}
\hline & $\begin{array}{c}\text { Personen- } \\
\text { anteil }\end{array}$ & $\begin{array}{c}\text { Mengen- } \\
\text { anteil }\end{array}$ & $\begin{array}{c}\text { Wert- } \\
\text { anteil }\end{array}$ & $\begin{array}{c}\text { Durch- } \\
\text { schnitts-preis }\end{array}$ \\
\hline $\begin{array}{l}\text { Discounter- } \\
\text { Kunden }\end{array}$ & $21 \%$ & $12 \%$ & $7 \%$ & 2,82 \\
\hline LEH-Kunden & $27 \%$ & $12 \%$ & $8 \%$ & 3,43 \\
\hline $\begin{array}{l}\text { Supermarkt- } \\
\text { Kunden }\end{array}$ & $18 \%$ & $13 \%$ & $10 \%$ & 3,87 \\
\hline Ab Hof-Kunden & $9 \%$ & $23 \%$ & $24 \%$ & 5,48 \\
\hline $\begin{array}{l}\text { Fachgeschäft- } \\
\text { Kunden }\end{array}$ & $6 \%$ & $17 \%$ & $27 \%$ & 8,96 \\
\hline $\begin{array}{l}\text { Multichannel- } \\
\text { Kunden }\end{array}$ & $19 \%$ & $22 \%$ & $24 \%$ & 6,02 \\
\hline
\end{tabular}

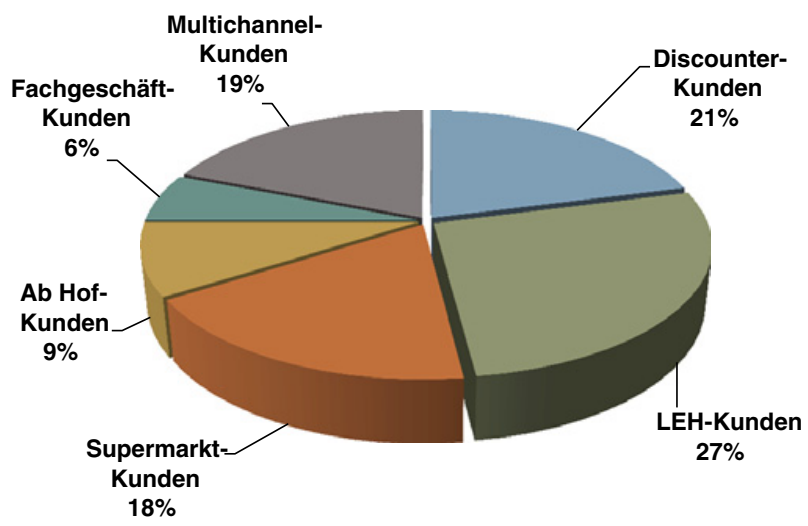

Abb 3. Größe der Einkaufsstätten-Segmente.

"Discount- und LEH-Weinkunden" waren jünger, besaßen eine einfachere schulische Bildung, gehörten zu einem deutlich höheren Anteil den unteren Einkommensgruppen an und wohnten mehr im Nordwesten, in NRW, Bayern sowie in den neuen Bundesländern, Berlin ausgenommen.

Dagegen entsprachen "Supermarktkunden" eher dem Durchschnitt der Bevölkerung über 16 Jahren und damit allen Weintrinkern und machten einen etwas höheren Anteil in den gehobenen Einkommensgruppen aus.

Die Gruppe der "Ab-Hof-Weinkunden" besaß den mit Abstand höchsten Anteil an Personen über 65 Jahre, eher eine durchschnittliche Bildungsstruktur und ein durchschnittliches Einkommen und wurden vor allem in der Region Mitte-West (Hessen, Rheinland-Pfalz und im Saarland) angetroffen.

Vertreter der Rubrik "Fachhandelskunden" besaßen ein höheres Einkommen und lebten verstärkt in den großen Städten.

Demgegenüber zeigten "Multichannel-Weinkunden" ein markant abweichendes soziodemografisches Profil mit Personen eher mittleren Alters, gehobenem Bildungsniveau und hohem Einkommen. Diese lebten eher im Südwesten Deutschlands.

\section{Fazit}

Um den Weinmarkt detailliert und vielseitig beschreiben zu können, müssen die Konsumenten eines Marktes mit hilfe mehrerer Segmentierungsmodelle analysiert werden. 
Die vollständige Version dieser Studie enthält insgesamt neun Segmentierungsmodelle (segmentiert nach Geschlecht, Alter, Bundesländern, Trinkhäufigkeit, Involvement, Geschmackspräferenz, Farbintensität, Herkunftspräferenz, sozialer Klassen und Einkaufsstätten-Nutzu ng), welche die Möglichkeit anbieten, die passenden Zielgruppen auszuwählen und die Weinkonsumenten identifizieren zu können.

\section{References}

[1] L. Agnoli, D. Begalli, R. Capitello. IJWBR, 2, 176-192 (2011)

[2] M. Alebaki, O. Iakovidou. New Medit, 4, 31-40 (2010)

[3] J-M. Aurifeille, P.G. Quester, L. Lockshin, T. Spawton. Int. Mark. Rev, 4, 369-386 (2002)

[4] P. Berni, D. Begalli, R. Capitello. Journal of International Food \& Agribusiness Marketing, 1, 117-145 (2005)

[5] T. A. Brunner, M. Siegrist. Brit Food J, 3, 353-373 (2011)

[6] J. Bruwer, E. Li, M. Reid. Journal of Wine Research, 3, 217-242 (2002)

[7] J. Bruwer, E. Li. Journal of Wine Research, 1, 19-34 (2007)

[8] L. Casini, C. Seghieri, F. Torrisi. Paper presented on the 3rd International Wine Business Research Conference, Montpellier (F) (2006)

[9] J.S. Dubow. J. Advertising Res, 2, 11-18 (1992)

[10] J. Fountain, C. Lamb. IJWBR, 2, 107-124 (2011)

[11] S. Geraghty, A. Torres. IJWBR, 2, 143-154 (2009)

[12] S. Goodman, L. Lockshin, E. Cohen. Revue Internationale des Sciences Sociales (Marketing and Communication, Market Management), 1, 94-112 (2008)

[13] S. Goodman, L. Lockshin, H. Remaud. Paper presented at the 5th Academy of Wine Business Research Conference, Auckland (NZ) (2010)

[14] J. Hall, M. Winchester. IJWM, 1, 19-35 (1999)

[15] J. Hall, W. Binney, G.B. O’ Mahony. IJWM, 3, 29-43 (2004)
[16] J. Hall, M. Shaw, L. Doole. IJWM, 2/3, 83-92 (1997)

[17] A. Hofmeister, G. Totth. Paper presented at the $1^{\text {st }}$ Academy of Wine Business Research Conference, Adelaide (AUS) (2003)

[18] L. W. Johnson, L. Ringham, K. Jurd. IJWM, 1, 26-31 (1991)

[19] T. Johnson, J. Bruwer. IJWM, 1, 5-33 (2003)

[20] Z. Kalazić, M.L. Šimić, J. Horvat. J Food Prod Market, 3, 325-335 (2010)

[21] N. Kolyesnikova, T. H. Dodd, D. F. Duhan. IJWBR, 4, 321-334 (2008)

[22] L. Lockshin, P. Quester, T. Spawton. Journal of Wine Research, 3, 223-236 (2001)

[23] L. Lockshin, T. Spawton, G. Macintosh. Journal of Retailing and Consumer Service, 3, 171-183 (1997)

[24] L. Lockshin, E. Cohen. Eur. J. Marketing, 7/8, 1236-1252 (2009)

[25] M. McGarry, S. Carpernter, E. Qenani-Petrela. JFDRS, 1, 186-91 (2005)

[26] D. McKinna in P. Hayes (Ed.), Proceedings of the Conference Grapes and Wine - The business end, Victorian Wine Industry Association, Melbourne (1987)

[27] S. Mueller, G. Szolnoki. Food Qual Prefer, 21, 774-783 (2010)

[28] S. Mueller, H. Remaud, Y. Chabin. IJWBR, 2, 124-144 (2011)

[29] J.E. Olsen, L. Thach, L. Nowak. Journal of Wine Research, 1, 1-18 (2007)

[30] P.G. Quester, J. Smart. IJWM, 3, 37-56 (1996)

[31] A. Riviezzo, A. De Nisco, A. Garofano. Paper presented at the 6th Academy of Wine Business Research Conference, Bordeaux (F) (2011)

[32] K. Simpson, P. Bretherton in K.A. Smith, C. Schott (Eds.). Proceedings of the New Zealand Tourism and Hospitality Research Conference, Wellington (NZ) 377-385 (2004)

[33] T. Spawton. Eur. J. Marketing, 3, 6-48 (1991)

[34] E.C. Thach, J.E. Olsen. Agribusiness, Special Issue on Wine Marketing, 307-322 (2006)

[35] A. Thomas, G. Pickering. Journal of Wine Research, 2/3, 127-138 (2003) 